\title{
Depression and anxiety in patients with chronic heart failure
}

\author{
Bruno Bordoni*,1, Fabiola Marelli2,3, Bruno Morabito ${ }^{2,3,4}$ \& Beatrice Sacconi ${ }^{4,5}$ \\ ${ }^{1}$ Foundation Don Carlo Gnocchi IRCCS, Department of Cardiology, Institute of Hospitalization \& Care with Scientific Address, S \\ Maria Nascente, Via Capecelatro 66, Milan 20100, Italy \\ ${ }^{2}$ CRESO, School of Osteopathic Centre for Research \& Studies, Gorla Minore (VA) Piazza XXV Aprile 4, 21055, Italy \\ ${ }^{3}$ CRESO, School of Osteopathic Centre for Research \& Studies, Via Fanella, 9161032 Fano (Pesaro Urbino), Italy \\ ${ }^{4}$ Department of Radiological, Oncological \& Anatomopathological Sciences, Sapienza University of Rome, Viale Regina Elena 324, \\ 00161, Rome, Italy \\ ${ }^{5}$ Center for Life Nano Science@Sapienza, Istituto Italiano di Tecnologia, Viale Regina Elena 291, Rome, Italy \\ * Author for correspondence: bordonibruno@hotmail.com
}

\section{"In the field of respiratory and cardiac rehabilitation, it has been demonstrated that an improvement of the respiratory activity can lead to an improved emotional status"”}

First draft submitted: 3 August 2017; Accepted for publication: 9 November 2017; Published online: 22 January 2018

Keywords: anxiety $\bullet$ baroreceptors $\bullet$ chronic heart failure $\bullet$ diaphragm • depression $\bullet$ inflammatory cytokines • phrenic nerve $\bullet$ psychiatric disorders $\bullet$ rehabilitation $\bullet$ vagus nerve

Chronic heart failure (CHF) is a progressive, debilitating disease, resulting in a decline in the quality of life of the patients and in very high social economic costs. CHF is a complex disease, whose diagnosis is made based on physical examination of the patient by the physician, and based on medical history including symptoms such as congestion and/or hypoperfusion of the organs [1]. It is defined as the inability of the heart to supply the oxygen demands by the peripheral organs, causing negative effects on all the body systems [2]. Patients affected by CHF often have a low quality of life, and most of the patients have a short life expectancy, with high mortality rate within 5 years from diagnosis [3]. Current data estimate that 5.8 million people in America are currently affected, and about 23 million people worldwide. However, considering that the average age of the population is rising thanks to advances in the medical treatment for cardiovascular diseases, the number of patients diagnosed with $\mathrm{CHF}$ is expected to increase in the coming years [4]. Fatigue and intolerance to exercise are the most obvious clinical manifestations in patients with CHF [5]. Other comorbidities are commonly observed, such as depression and anxiety [6].

The focus of the present article is to discuss the potential correlation between the above-mentioned comorbidities, $\mathrm{CHF}$ and the diaphragmatic innervation and functionality. It has been demonstrated that some psychiatric behaviors can have impact on the survival rate, as discussed in the paragraphs below. In this article the authors hypothesize that the dysfunction of the diaphragm often detectable in this chronic condition can have a role in the occurrence of these comorbidities. In the field of respiratory and cardiac rehabilitation, it has been demonstrated that an improvement of the respiratory activity can lead to an improved emotional status [7].

\section{Adaptation of the diaphragm in patients affected by CHF}

The diaphragm is innervated by the phrenic nerve and the vagus nerve (in the portion which constitutes the esophageal hiatus) [8].

The inspiratory dysfunction of the diaphragm is a key element in patients with CHF, influencing mortality and morbidity rates [9]. Diaphragmatic abnormalities appear earlier than those related to the lower limbs of the patients. The breath becomes more superficial, with increase of the sympathetic activity; the sympathetic nervous system stimulates the phrenic afferents of group IV, which in turn stimulate the sympathetic fibers, in a vicious circle. This pathological condition predisposes the patient to arrhythmias and higher risk of sudden death [9]. The phrenic nerve experiences a dyssynergia between the transmitted pulse and the correspondent strength, resulting 
in degeneration and denervation of the synaptic plaque. In the diaphragm of these patients, a decrease of the contractile mass is observed, especially in the amount of titin, resulting in decreased elasticity and in the percentage of myosin, negatively affecting the strength $[9,10]$.

The exercise intolerance in patients with CHF does not correlate with the common functional index (ejection frequency); rather it is the peripheral muscle adaptation, including that of the diaphragm, to have a heavy influence on the symptomatic scenario $[11,12]$.

\section{Depression \& anxiety in CHF}

Psychiatric disorders such as depression and anxiety, are symptomatic scenarios often observed in patients with CHF [6]. These comorbidities complicate the therapeutic approach and increase hospitalizations and mortality rate $[13,14]$. Depression affects at least one-fifth of patients with CHF, worsening the NYHA class; depression affects these patients with from two- to three-times more than the general population [13]. The real correlation of the depression with CHF is not completely clear, despite some similarity in the physiopathological mechanisms, such as high levels of catecholamines, cortisol and inflammatory cytokines (IL-6, IL-1 $\beta$, TNF- $\alpha$ ), whose presence highlights the clinical and prognostic significance of depression [13]. Depression negatively affects the quality of life and functional status of the patient, decreasing the level of physical activity and worsening the survival rate. A definitive consensus on the best pharmacological therapy for the treatment of depression in CHF has not been reached; furthermore, data on the incidence of depression in this patients' class are probably underestimated [13].

Anxiety is another often overlooked symptom, increasing the hospitalization and mortality rates [14]. Anxiety can negatively affect the quality of the breath, creating panic and chest pain, and exacerbating the symptoms of CHF [14]. The current literature does not exhaustively discuss the reasons for the presence of the anxiety; anyway, anxiety can be probably correlated with a dysfunction of the hypothalamic-pituitary-adrenal axis, usually observed even in CHF [14]. No reliable data are available on the incidence of anxiety in this patients population, as well as on the pathophysiological mechanisms causing its presence in subjects with CHF.

\section{Phrenic \& vagus nerve: functions}

The phrenic and vagus nerves are involved in the respiratory functions of the diaphragm, in perfect synergy. The diaphragm influences the emotional status.

During this respiratory action, the systolic pressure increases with a decrease in the cardiac frequency [15]. We know that, when the baroreceptor located in the carotid body and in the area of the aortic arch (in the adventitia of the vessels) are stimulated by the cardiac cycle (in particular during the systolic phase). The afferent fibers from baroreceptor reach the nucleus of the solitary tract (NTS), which regulates the efferent activation to the vagal system and the sympathetic inhibitory efferents at the spinal level in the nucleus ambiguous, the dorsal motor nucleus and the rostral ventrolateral area of the medulla [16]. The baroreceptorial afferents influence different areas of the CNS, with a general inhibitory effect. The NTS is interconnected with the reticular formation, and then to the lateral, medial and prefrontal insula, and to the anterior cingulate cortex; also the thalamus, hypothalamus and periaqueductal gray area receive baroreceptorial signals from NTS. There is a close relationship between the emotions, the breath and the intervention of baroreceptors [17].

Emotional conditions such as anxiety and depression can negatively affect the baroreceptors' response, as well as they can produce an altered function on the diaphragm. The action of the diaphragm is not only controlled by metabolic factors, but also by emotional states such as sadness, fear, anxiety and anger. The interaction between the breath and the emotions involves a complex interaction between the brainstem and a few brain centers such as the limbic area and the cortex [18]. The amygdala, which is part of the limbic system, is connected in biunivocal way to the respiratory areas, such as medulla, and it is considered the most important area in managing the emotional breath. The amygdala is stimulated by a dopaminergic production of the tegmental area of the midbrain, and recent studies on animal models suggest that the dopamine that reaches the amygdala regulates the emotional breath. The efferents derived from the amygdala pass through the areas related to the breath, such as the NTS and other correlated structures $[19,20]$.

The breath stimulates the mechanoreceptors of the diaphragm and the visceroceptors of the viscera moving during the respiration, which constitute the interoception mechanism. This is the awareness of the body condition based on the information derived directly from the body. The afferent fibers of the interoception are connected with the autonomic and homeostatic centers of the spinal cord and of the brainstem, and then with the anterior cingulate cortex and with the dorsal posterior insula, through the thalamus-cortical tract [21]. The interoception can modulate 
the exteroceptive representation of the body; a dysregulation in the interoceptive pathways could cause a distortion of the body image, affecting the emotional condition. Anxiety can alter a few afferent pathways related to breathing, amplifying one or more receptor pathways related to the respiration (quickly and slowly adapting receptors, type-C bronchial and lung receptors, A-delta-type receptors, cough receptors and neuroepithelial receptors) [22].

\section{New hypotheses \& considerations on psychiatric status}

The phrenic and vagus nerves provide visceral information. A current hypothesis is related to the solicitation of visceral nociceptors, sending continuous afferents in response to mechanical stress. This peripheral solicitation could lead to a central and spinal awareness, establishing a vicious circle between visceral information and emotional status [23]. The visceral afferents, both interoceptive and nociceptive (small caliber A-delta and C afferents) reach the lamina I and II of the spinal cord, and are connected to some supraspinal centers, such as the ventromedial posterior nucleus of the thalamus and the limbic area [24,25]. The phrenic and vagus nerves could be stimulated and determine abnormal afferents, or being submitted to efferents derived from the supraspinal centers stimulated by the nociceptive information of the mediastinal viscera.

The peripheral nerve structure is subjected to daily mechanical stress, such as when a joint moves, with alternance of compression and stretching. Compression happens when the surrounding tissues create a longitudinal force to a nerve, such as when the muscles are stretched, while stretching occurs when a force is parallel to the nerve, for example, when the elbow is flexed, the ulnar nerve is stretched [26]. The sliding of the fascial structures of the nerve and the nerve slippage between the various tissues is fundamental, so that the mechanical stress can communicate properly with the ability of adaptation and regeneration of the nerve. Abnormalities will lead to dysfunction and pathology [26]. Where there is a nerve impingment, the rigidity of its fascial structures increases during articular or respiratory movements; the nerve may undergo a reduction in its diameter, defined as transverse contraction, with an increase in the pressure of the endoneural compartment. Repetitive elongations of nerves with reduced elasticity of the fascial properties will induce additional inability in the nerve's sliding, decreasing the blood flow and leading to potential ischemic processes [26]. The fascial structures become more sensitive to mechanical stimuli and, after a few days of local inflammation, are able to generate an action potential similar to the initial stimulus causing the dysfunction; this potential can have an anterograde and retrograde propagation, causing inflammation at the extremities of the neural tract, such as in the spinal cord and in the innervated tissues. This mechanism is called ectopic electrogenesis [26]. In patients with CHF, the diaphragm muscle shows abnormalities, with changes in morphology and function, position and metabolism. The vagus and the phrenic nerves can undergo axonal alterations if the muscle movement is limited for such dysfunction, over time resulting in modified awareness schemes and allodynia [26,27].

The baroreceptor system, which influences emotional state, is altered in patients with CHF [11]. Most likely, the pathological changes observed for the diaphragm negatively affect its innervation, causing a baroreceptor dysfunction. This could be a further cause for psychiatric conditions. We can still speculate that an altered function of the diaphragm may adversely influence the patient's emotional state, probably because the interoceptive mechanisms stimulated by breathing are handled as motivational information, since these are bidirectional pathways [18]. The breathing requested during physical exercise could cause strong emotional reactions in anxious people, worsening the respiratory function [15]. The interoception is also related to visceral movement during respiration; people more susceptible to visceral afferents usually show more intense emotions. A potential cause might be related to neurogenic neuroinflammation in the spinal cord, where different areas are more likely to respond to minimal stimuli, leading to higher levels of anxiety and pain [18]. This event could lead to a pleiotropic effect of functional impairment of the muscle tissue, further destabilizing the function of the diphragm. The term of 'emotional respiratory allodynia' can be used when the breath, stimulating the interoceptive afferent pathways, causes psychological symptoms [15].

The innervation of the diaphragm muscle may be directly responsible for the emotional state of the patient through the phrenic nerve, not only due to the interoceptive mechanism. The afferent stimulation to the NTS by the phrenic nerve could affect the emotional response, because NTS handles the visceral afferents and has a close relationship with the nerve [28]. The phrenic nerve forms subdiaphragmatic ganglia and is connected with the adrenal gland; anyway, further data even on these connections are currently needed. We know that the adrenal gland and the hypothalamus-pituitary axis affect the intensity of the emotions [29]. It should be useful to further investigate the potential relation between the hypothalamus-pituitary axis, the phrenic nerve's activity and the emotional status. The vagus nerve affects the emotional spectrum and the respiratory rhythm, probably 
always through NTS. Anyway, reliable correlations are not known, neither if there are bi-directional mechanisms influencing the emotions and involving the diaphragm portions innervated by the vagus nerve [30].

\section{Conclusion}

CHF is a progressive, debilitating disease, defined as the inability of the heart to supply the oxygen demands by the peripheral organs. The article reviews the available information on the potential role of the diaphragm in this disease and emotional symptoms.

The close relationship between morphology and function of the diaphragm, the connections between the vagus and the phrenic nerves, as well as their collaboration for the diaphragm function, lead us to suggest that a dyssynergia between the two nerves could cause emotional pathological behavior. The management in these medical conditions should always be multidisciplinary, in order to have a global vision of the patient.

Over the next 5-10 years in the clinical and rehabilitative field, more attention will be given to diaphragm muscle, thanks to research that will further demonstrate the importance of the patient's breathing and symptomatic connection.

Financial \& competing interests disclosure

The authors have no relevant affiliations or financial involvement with any organization or entity with a financial interest in or financial conflict with the subject matter or materials discussed in the manuscript. This includes employment, consultancies, honoraria, stock ownership or options, expert testimony, grants or patents received or pending, or royalties.

No writing assistance was utilized in the production of this manuscript.

\section{Open access}

This work is licensed under the Attribution-NonCommercial-NoDerivatives 4.0 Unported License. To view a copy of this license, visit http://creativecommons.org/licenses/by-nc-nd/4.0/

\section{References}

1. Ramani GV, Uber PA, Mehra MR. Chronic heart failure: contemporary diagnosis and management. Mayo Clin. Proc. 85(2), 180-195 (2010).

2. Tzanis G, Dimopoulos S, Agapitou V, Nanas S. Exercise intolerance in chronic heart failure: the role of cortisol and the catabolic state. Curr. Heart Fail. Rep. 11(1), 70-79 (2014).

3. Siabani S, Driscoll T, Davidson PM, Leeder SR. A randomized controlled trial to evaluate an educational strategy involving community health volunteers in improving self-care in patients with chronic heart failure: rationale, design and methodology. SpringerPlus 3, 689 (2014).

4. Gaggin HK, Januzzi JL Jr. Biomarkers and diagnostics in heart failure. Biochim. Biophys. Acta. 1832(12), 2442-2450 (2013).

5. Bordoni B, Marelli F. The fascial system and exercise intolerance in patients with chronic heart failure: hypothesis of osteopathic treatment. J. Multidiscip. Healthc. 8, 489-494 (2015).

6. Janssen DJ, Spruit MA, Uszko-Lencer NH, Schols JM, Wouters EF. Symptoms, comorbidities, and health care in advanced chronic obstructive pulmonary disease or chronic heart failure. J. Palliat. Med. 14(6), 735-743 (2011).

7. Louvaris Z, Vogiatzis I. Physiological basis of cardiopulmonary rehabilitation in patients with lung or heart disease. Breathe (Sheff) 11(2), $120-127$ (2015).

8. Bordoni B, Zanier E. Anatomic connections of the diaphragm: influence of respiration on the body system. J. Multidiscip. Healthc. 6, 281-291 (2013).

9. Kelley RC, Ferreira LF. Diaphragm abnormalities in heart failure and aging: mechanisms and integration of cardiovascular and respiratory pathophysiology. Heart Fail. Rev. 22(2), 191-207 (2016).

10. van Hees HW, van der Heijden HF, Ottenheijm CA et al. Diaphragm single-fiber weakness and loss of myosin in congestive heart failure rats. Am. J. Physiol. Heart Circ. Physiol. 293(1), H819-H828 (2007).

11. Vogiatzis I, Zakynthinos S. The physiological basis of rehabilitation in chronic heart and lung disease. J. Appl. Physiol. (1985) 115(1), 16-21 (2013).

12. Evans RA, Singh SJ, Collier R, Loke I, Steiner MC, Morgan MD. Generic, symptom based, exercise rehabilitation; integrating patients with COPD and heart failure. Respir. Med. 104(10), 1473-1481 (2010).

13. DeJongh B, Birkeland K, Brenner M. Managing comorbidities in patients with chronic heart failure: first, do no harm. Am. J. Cardiovasc. Drugs 15(3), 171-184 (2015).

14. Vongmany J, Hickman LD, Lewis J, Newton PJ, Phillips JL. Anxiety in chronic heart failure and the risk of increased hospitalisations and mortality: a systematic review. Eur. J. Cardiovasc. Nurs. 15(7), 478-485 (2016). 
15. Bordoni B, Marelli F, Bordoni G. A review of analgesic and emotive breathing: a multidisciplinary approach. J. Multidiscip. Healthc. 9 , 97-102 (2016).

16. Reyes del Paso GA, Montoro C, Muñóz Ladrón de Guevara C, Duschek S, Jennings JR. The effect of baroreceptor stimulation on pain perception depends on the elicitation of the reflex cardiovascular response: evidence of the interplay between the two branches of the baroreceptor system. Biol. Psychol. 101, 82-90 (2014).

17. Herman JP. Regulation of hypothalamo-pituitary-adrenocortical responses to stressors by the nucleus of the solitary tract/dorsal vagal complex. Cell Mol. Neurobiol. doi:10.1007/s10571-017-0543-8 (2017) (Epub ahead of print).

18. Bordoni B, Marelli F, Morabito B, Sacconi B. Depression, anxiety and chronic pain in patients with chronic obstructive pulmonary disease: the influence of breath. Monaldi Arch. Chest. Dis. 87(1), 811 (2017).

19. Buff $\mathrm{C}$, Brinkmann L, Bruchmann $\mathrm{M}$ et al. Activity alterations in the bed nucleus of the stria terminalis and amygdala during threat anticipation in generalized anxiety disorder. Soc. Cogn. Affect. Neurosci. doi:10.1093/scan/nsx103 (2017) (Epub ahead of print).

20. Sharp PB, Telzer EH. Structural connectomics of anxious arousal in early adolescence: translating clinical and ethological findings. Neuroimage Clin. 16, 604-609 (2017).

21. Bordoni B, Marelli F. Emotions in motion: myofascial interoception. Complement. Med. Res. 24(2), 110-113 (2017).

22. Paulus MP. The breathing conundrum-interoceptive sensitivity and anxiety. Depress. Anxiety 30(4), 315-320 (2013).

23. Greenwood-van Meerveld B, Moloney RD, Johnson AC, Vicario M. Mechanisms of stress-induced visceral pain: implications in irritable bowel syndrome. J. Neuroendocrinol. 28(8) doi:10.1111/jne.12361 (2016).

24. Hong JY, Naliboff B, Labus JS et al. Altered brain responses in subjects with irritable bowel syndrome during cued and uncued pain expectation. Neurogastroenterol. Motil. 28(1), 127-138 (2016).

25. Jänig W. Mechanical allodynia generated by stimulation of unmyelinated afferent nerve fibres. J. Physiol. 589(Pt 18), 4407-4408 (2011).

26. Bordoni B, Bordoni G. Reflections on osteopathic fascia treatment in the peripheral nervous system. J. Pain Res. 8, 735-740 (2015).

27. Bordoni B, Marelli F. Failed back surgery syndrome: review and new hypotheses. J. Pain Res. 9, 17-22 (2016).

28. McDougall SJ, Andresen MC. Independent transmission of convergent visceral primary afferents in the solitary tract nucleus. $J$. Neurophysiol. 109(2), 507-517 (2013).

29. Tubbs RS, Stetler W, Kelly DR et al. Immunohistochemical study of the phrenic ganglion. Anat. Sci. Int. 83(3), 159-161 (2008).

30. Bodenlos JS, Kose S, Borckardt JJ et al. Vagus nerve stimulation and emotional responses to food among depressed patients. J. Diabetes Sci. Technol. 1(5), 771-779 (2007). 
